\title{
Exploring the needs and perceptions regarding healthy eating among people at risk of food insecurity: a qualitative analysis
}

\section{Abstract}

Introduction: Healthy eating behaviors are essential determinants of overall health. These behaviors are generally poor among people at risk of experiencing food insecurity, which may be caused by many factors including perceived higher costs of healthy foods, financial stress, inadequate nutrition knowledge, and inadequate skills required for healthy food preparation. Few studies have examined how these factors influence eating behaviors among people at risk of experiencing food insecurity. We therefore aimed to gain a better understanding of the needs and perceptions regarding healthy eating in this target group.

Methods: We conducted a qualitative open interview study with 10 participants at risk of experiencing food insecurity. Thematic analysis identified four core themes on factors influencing healthy eating behaviors: (1) health related topics, influences on eating behaviors by both the (2) social and (3) physical environment, and (4) financial influences on eating behaviors.

Results: Overall, participants showed adequate nutrition knowledge. However, eating behaviors were strongly influenced by both social environmental factors (e.g. child food preferences and cultural food habits), and physical environmental factors (e.g. temptations in the local food environment). Perceived barriers for healthy eating behaviors included poor mental health, financial stress, and high prices of healthy foods. Participants had a generally positive and conscious attitude towards their financial situation, among others reflected in their strategies to cope with a limited budget. Food insecurity was mostly mentioned in reference to the past or to others and not to participants' own current experiences. Participants were familiar with several existing resources to reduce food-related financial strain and generally had a positive attitude towards these resources. An exception was the Food Bank, which was highly criticized on its food parcel content. Proposed new resources included distributing free meals, facilitating social contacts, increasing healthy food supply in the neighborhood, and lowering prices of healthy foods.

Conclusion: The insights from this study increase understanding of factors influencing healthy eating behaviors of people at risk of food insecurity. Therefore, this study could inform future development of potential interventions aiming at helping people at risk of experiencing food insecurity to improve healthy eating, thereby decreasing the risk of diet-related diseases.

\section{Conflict of Interest}

There is no conflict of interest 